\title{
Characterizing a CT esophagram protocol after flexible endoscopic diverticulotomy for Zenker's diverticulum: a retrospective series
}

\author{
Debdeep Banerjee ${ }^{1}$, Laura L. Magnelli ${ }^{2}$, Mailin Oliva ${ }^{3}$, Neha Malik ${ }^{3}$, Brittney M. Ginsburg ${ }^{1}$, \\ Joseph R. Grajo ${ }^{2}$, Tony S. Brar ${ }^{4}$, Donevan R. Westerveld ${ }^{1}$, Dennis Yang ${ }^{4}$, Peter V. Draganov ${ }^{4}$
}

${ }^{1}$ Department of Medicine, University of Florida, Gainesville, FL, USA; ${ }^{2}$ Division of Abdominal Imaging, Department of Radiology, University of Florida, Gainesville, FL, USA; ${ }^{3}$ College of Medicine, University of Florida, Gainesville, FL, USA; ${ }^{4}$ Division of Gastroenterology, Hepatology and Nutrition, Department of Medicine, University of Florida, Gainesville, FL, USA

Contributions: (I) Conception and design: D Banerjee, JR Grajo, D Yang, PV Draganov; (II) Administrative support: D Banerjee, TS Brar, DR Westerveld, PV Draganov; (III) Provision of study materials or patients: LL Magnelli, TS Brar, JR Grajo, D Yang, PV Draganov; (IV) Collection and assembly of data: D Banerjee, LL Magnelli, M Oliva, N Malik, BM Ginsburg; (V) Data analysis and interpretation: D Banerjee, LL Magnelli, JR Grajo, D Yang, PV Draganov; (VI) Manuscript writing: All authors; (VII) Final approval of manuscript: All authors.

Correspondence to: Peter V. Draganov, MD. Professor of Medicine, Division of Gastroenterology, Hepatology, and Nutrition, University of Florida, 1329 SW 16th Street, Gainesville, FL 32608, USA. Email: peter.draganov@medicine.ufl.edu.

Background: Flexible endoscopic cricopharyngeal myotomy and septotomy offer a minimally invasive transluminal option for the treatment of symptomatic Zenker's diverticulum (ZD). There is currently no consensus regarding postoperative follow-up imaging. We suggest a standardized computed tomography (CT) esophagram protocol for radiographic evaluation of postoperative findings.

Methods: Single center retrospective analysis of patients with symptomatic ZD who underwent flexible endoscopic diverticulotomy and postoperative imaging with CT esophagram from January 2015 to March 2020. An experienced radiologist blinded to the initial imaging reports prospectively interpreted all CT esophagram findings, in order to minimize bias.

Results: Twenty-one patients underwent CT esophagram following flexible endoscopic diverticulotomy for ZD. Diverticulotomy was technically successful in all patients. Most common findings on imaging included: atelectasis $(13 / 21 ; 62 \%)$, persistent esophageal diverticulum $(7 / 21 ; 33 \%)$, pneumomediastinum $(3 / 21 ; 14 \%)$, aspiration $(2 / 21 ; 10 \%)$, and extraluminal air and contrast extravasation consistent with focal esophageal perforation $(1 / 21 ; 5 \%)$.

Conclusions: We describe a standardized, simple, and accessible CT esophagram protocol for postoperative imaging of patients with post-flexible endoscopic cricopharyngeal myotomy and septotomy for ZD. CT esophagram facilitates a definitive exclusion of focal esophageal perforation as a postoperative complication of flexible endoscopic diverticulotomy by ruling out extraluminal air and contrast extravasation.

Keywords: Zenker's diverticulum (ZD); esophagram; endoscopy; septotomy; diverticulotomy

Received: 28 July 2020; Accepted: 13 September 2020; Published: 25 October 2022.

doi: $10.21037 / \operatorname{tgh}-20-269$

View this article at: http://dx.doi.org/10.21037/tgh-20-269

$\wedge$ ORCID: 0000-0002-5154-1822. 


\section{Introduction}

Zenker's diverticulum (ZD) is a hypopharyngeal acquired anatomic defect that may affect quality of life by causing classic symptoms of cough, dysphagia, food trapping, regurgitation, and weight loss. Previously managed almost exclusively through open surgery or transoral rigid stapled diverticulotomy, now symptomatic ZD is increasingly treated via flexible endoscopic cricopharyngeal myotomy and septotomy (a.k.a. diverticulotomy) (1-4). Flexible endoscopic diverticulotomy offers a minimally invasive transluminal approach for ZD intervention through a natural orifice and has been associated with high symptomatic response rate and low incidence of adverse events (5). Nonetheless, serious adverse events, most frequently related to perforation, can occur, which may lead to abscess formation, mediastinitis, and bronchoesophageal fistulas $(6,7)$. Common modalities used to detect perforation include water-soluble or barium swallow esophagrams, but objective findings on these traditional exams correlate poorly with clinical symptoms such as fever, neck pain, and dysphagia (8-10). Therefore, true perforation detected on post-intervention imaging can allow for earlier diagnosis and intervention.

Computed tomography (CT) esophagram involves imaging of the neck, chest, and upper abdomen with the administration of oral contrast medium. This imaging modality has been used to evaluate for leak after peroral endoscopic myotomy (POEM) procedure or when esophageal perforation is clinically suspected (post-trauma, post-surgical, foreign body ingestions) $(11,12)$. In POEM, postprocedural CT esophagram has been shown to offer several advantages. Firstly, utilizing oral contrast allows for a presumed greater sensitivity to detecting esophageal mucosal abnormalities as compared to video fluoroscopy. Secondly, by scanning multiple regions, abnormalities in the neck, mediastinum, lungs, and upper abdomen may be detected. Thirdly, unlike video fluoroscopy, CT esophagram does not require the presence of a radiologist to perform the study, which may potentially increase availability of imaging at all hours (11). However, data on the performance of CT esophagram for postoperative imaging of other conditions is limited. In this study, we report the radiographic findings on CT esophagram in patients following flexible endoscopic cricopharyngeal myotomy and septotomy for symptomatic ZD. We present the following article in accordance with the STROBE reporting checklist (available at https://tgh. amegroups.com/article/view/10.21037/tgh-20-269/rc).

\section{Methods}

\section{Patient population}

The study was conducted in accordance with the Declaration of Helsinki (as revised in 2013). The study was approved by the Institutional Review Board at the University of Florida (IRB202001012). This was a retrospective cohort study, and all patients signed informed consent for the procedure prior to therapeutic endoscopy. Inclusion criteria involved: (I) patients $\geq 18$ years old with documented symptomatic ZD based on preoperative imaging and/or upper endoscopy; (II) patients who underwent flexible endoscopic cricopharyngeal myotomy for symptomatic ZD therapy; (III) flexible endoscopic procedures occurring between January 2015 and March 2020 for therapy of symptomatic ZD; (IV) patients receiving postoperative CT esophagram during the same hospital admission. All patients who received this endoscopic therapy for symptomatic ZD also obtained a postoperative CT esophagram; therefore, all patients that were treated were included. ZD length was measured based on baseline preoperative diagnostic imaging, which included video fluoroscopy, upper endoscopy, and CT.

\section{Flexible endoscopic diverticulotomy of $Z D$}

Patients were kept nil per os (NPO or nothing by mouth) on the day of the procedure. The procedure was done under general anesthesia. The endoscope was fitted with a distal attachment cap (Olympus America, Center Valley, Pennsylvania, USA) to better isolate the septum separating the true esophageal lumen from the diverticulum. Electrosurgical knives were used to incise the cricopharyngeal muscle and diverticular septum as previously described (13). The use of clips to approximate the incision edges at the conclusion of the diverticulotomy was performed at the discretion of the endoscopist. Patients were administered intravenous antibiotics (ampicillinsulbactam $1.5 \mathrm{~g}$ or ciprofloxacin $400 \mathrm{mg}$ ) intraoperatively and remained on oral prophylaxis following the procedure for a duration of 5 days total. All patients were admitted for overnight observation and postoperative CT esophagram.

\section{Postoperative CT esophagram}

Following endoscopy, patients were kept on strict NPO until CT esophagram was performed on either postoperative day (POD) 0 or 1 to exclude extraluminal contrast extravasation (i.e., esophageal perforation) and allow the safe transition 
to oral feeding. The CT esophagram consisted of a scan from the neck through the stomach before and after oral administration of 50 milliliters of iohexol divided into two portions, the second of which contained simply thick nectar. No intravenous contrast was given. Thin slice axial images were acquired with $1 \mathrm{~mm}$ reconstruction. Coronal and sagittal reconstructions were performed at the workstation (Visage Imaging, San Diego, California, USA). In order to minimize potential operator bias on existing radiologist interpretation summaries, a separate abdominal radiologist (L.L.M.) blinded to the initial imaging interpretations prospectively performed an independent evaluation of all CT esophagrams at the time of manuscript preparation (Figure 1). Severity of findings were described, as per the interpreting radiologist. Discrepancies were resolved by consensus, involving a second abdominal radiologist (J.R.G.), if necessary.

Diet was progressed to clear liquids on POD 1 if the CT esophagram was negative for extraluminal contrast extravasation. At the time of hospital discharge, patients were instructed to remain on a full liquid diet for 1 week before advancing to a mechanical soft diet for an additional week prior to finally advancing to a diet tolerated ad lib.

\section{Outcome measures}

The primary outcome was to characterize postoperative findings on CT esophagram following flexible endoscopic diverticulotomy for ZD. Findings on CT were considered clinically significant when a follow-up investigation or change in clinical care were triggered based on radiographic findings. Secondary outcomes included rate of technical success of the flexible endoscopic diverticulotomy, defined as the ability to complete flexible endoscopic diverticulotomy for ZD within one session. Adverse events were assessed based on previously established criteria by the American Society for Gastrointestinal Endoscopy (ASGE) (14).

\section{Statistical analysis}

Summary data were expressed as the mean \pm standard deviation (SD) for variables with a standard distribution, and the median and range for variables with a skewed distribution. Frequencies and percentages were calculated using basic descriptive statistics. Analysis was conducted using GraphPad Prism 8.3.0 for Windows (GraphPad Software, San Diego, California, USA).

\section{Results}

\section{Patient demographics}

A total of 21 patients ( $52 \%$ men; mean age $73 \pm 9.4$ years) with symptomatic ZD underwent flexible endoscopic diverticulotomy followed by postoperative CT esophagram during the study period. Median duration of symptoms prior to intervention was 36 months (range, $8-168$ months).

Most cases of ZD were diagnosed at baseline through video fluoroscopy or in combination with esophagogastroduodenoscopy (EGD) (19; 90\%). One patient had previously undergone POEM for type II achalasia in 2015. Two referred cases from otolaryngology had history of aborted attempts at transoral rigid stapled diverticulotomy due to a retrognathic mandible and an unfavorable distal location of the ZD, respectively (Table 1).

\section{Flexible endoscopic diverticulotomy of ZD}

Procedural characteristics are summarized in Table 2. The procedure was technically successful in $100 \%$ of the cases (Figure 2). $\mathrm{CO}_{2}$ insufflation was used in $100 \%$ of the cases. The mean total procedure time was $32 \pm 12.9$ minutes. Diverticulum length was described on preoperative baseline imaging in 13 patients with a median length of $25 \mathrm{~mm}$ (range, 15-60 mm). The scissors-type electrosurgical knives Clutch Cutter (DP2618DT-35 Fujifilm Endoscopy, Wayne, New Jersey, USA) and Stag Beetle (SB) (Olympus America, Center Valley, Pennsylvania, USA) were used in most cases (20; 95\%) (Table 2). One case used the SB Junior knife. In another case, both Clutch Cutter and Insulated tip type 2 (IT2) (Olympus America, Center Valley, Pennsylvania, USA) electrosurgical knives were utilized. Endoscopic clipping (mean 3 clips; SD 1.5; range, 0-6) to appose the edges of the diverticulotomy was performed in 20 patients (95\%). Nearly all $(20 / 21 ; 95 \%)$ were able to resume liquids by mouth on POD 0 or 1 in the absence of contrast extravasation/suspicion for leak on postoperative CT esophagram. The mean length of stay was $2 \pm 1.1$ days.

\section{Adverse events}

There were no immediate intraoperative or postoperative adverse events in 20 of 21 patients (95\%). One patient (a 61-year-old man) experienced throat pain and productive cough in addition to developing fever and tachycardia approximately 10 hours after diverticulotomy. No hemostatic clips had been placed at the conclusion of 


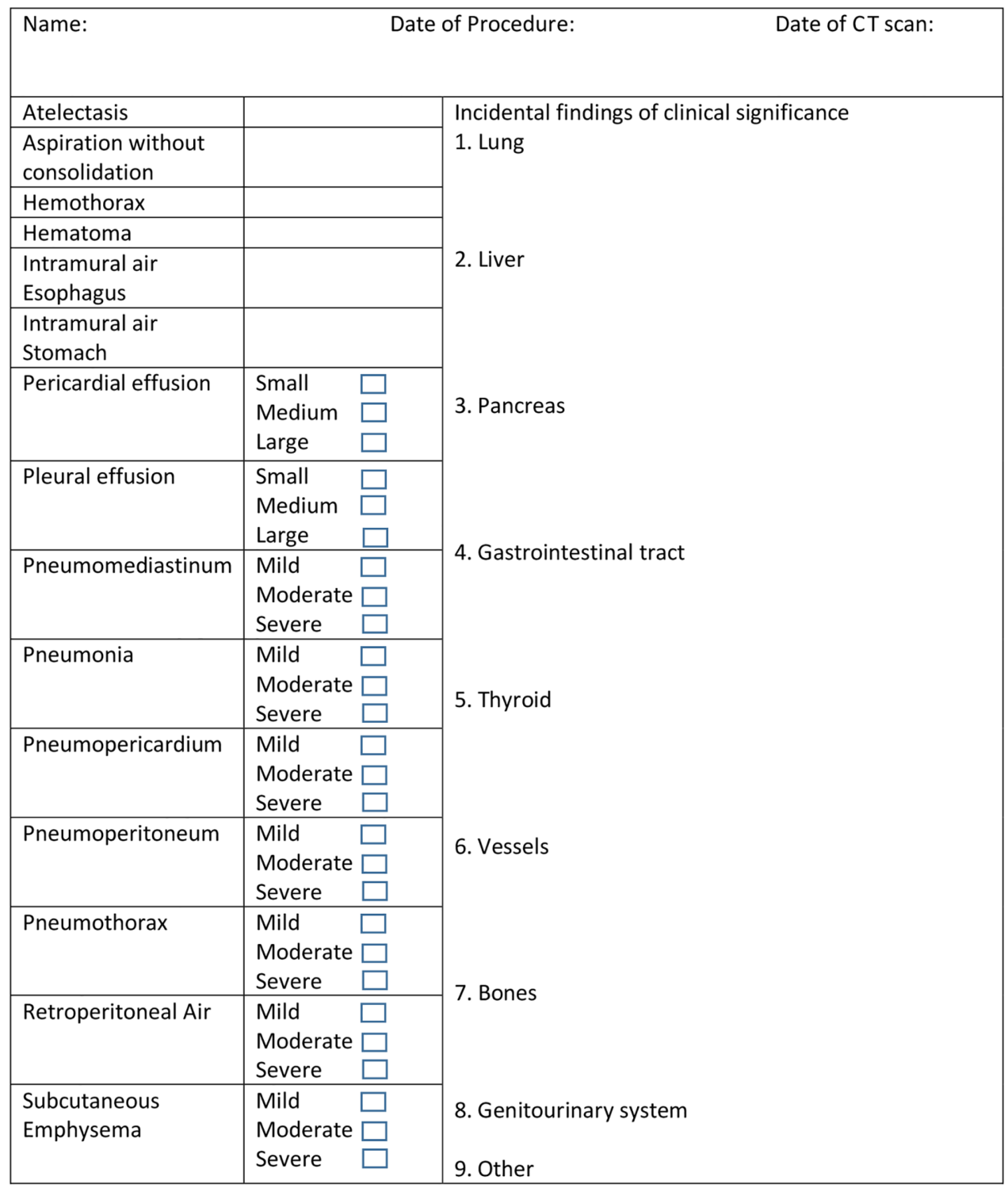

Figure 1 Computed tomography (CT) esophagram data collection form.

this procedure. Although no evidence of esophageal perforation was visible endoscopically during the procedure, extraluminal air and contrast extravasation were identified on postoperative POD $1 \mathrm{CT}$ esophagram (Figure 3) obtained 14 hours after endoscopy. The patient remained $\mathrm{NPO}$, received three days of intravenous antibiotics as part of a total 10-day antibiotic regimen, and underwent laparoscopic gastrostomy tube placement for nutritional support. Outpatient CT esophagram confirmed resolution of perforation, and the patient was able to resume oral diet 43 days after initial procedure.

\section{Postoperative CT esophagram findings}

All 21 patients underwent CT esophagram after flexible endoscopic diverticulotomy on POD $0(6 / 21 ; 29 \%)$ or on POD $1(15 / 21 ; 71 \%)$. The most common postoperative findings included: atelectasis $(13 / 21 ; 62 \%)$, residual esophageal diverticulum $(7 / 21 ; 33 \%)$, pulmonary infiltrate without consolidation $(3 / 21 ; 14 \%)$, pneumomediastinum $(3 / 21 ; 14 \%)$, and subcutaneous emphysema $(3 / 21 ; 14 \%)$. Other notable postoperative findings were relatively infrequent and mild, including aspiration $(2 / 21 ; 10 \%)$, 
Table 1 Baseline patient demographics (total $\mathrm{n}=21$ )

\begin{tabular}{lc}
\hline Characteristics & Number \\
\hline Age, mean \pm SD; years & $73 \pm 9.4$ \\
Gender, male: female & $11: 10$ \\
Symptom duration, median [range]; months & 36 [8-168] \\
Baseline diagnostic imaging for ZD & \\
Video fluoroscopy & $8(38 \%)$ \\
EGD & $3(14 \%)$ \\
CT & $1(5 \%)$ \\
Video fluoroscopy \& EGD & $8(38 \%)$ \\
Video fluoroscopy \& EGD \& CT & $1(5 \%)$ \\
Prior treatment for ZD & \\
None & $18(86 \%)$ \\
CO ${ }_{2}$ laser ablation & $1(5 \%)$ \\
Transoral rigid stapled diverticulotomy attempt & $2(10 \%)$ \\
\hline
\end{tabular}

SD, standard deviation; ZD, Zenker's diverticulum; EGD, esophagogastroduodenoscopy; CT, computed tomography; $\mathrm{CO}_{2}$, carbon dioxide.

pneumothorax $(1 / 21 ; 5 \%)$, and pneumoperitoneum (1/21; $5 \%)$. Aspiration was defined as visualization of contrast entering the subglottic lower respiratory tract (15). Pleural effusion and pericardial effusion were described in $2 / 21$ or $10 \%$ of patients each, respectively. As previously noted, extraluminal air and contrast extravasation was identified in one patient (Table 3). Other incidental findings on CT esophagram were referred for follow-up with each patient's primary care provider (Table 4). One patient had findings on inpatient postoperative CT esophagram of both pneumothorax and pneumomediastinum. CT esophagram on POD 1 had revealed a tiny left apical pneumothorax and mild pneumoperitoneum, pneumomediastinum, and subcutaneous emphysema. These were considered procedure-related findings and may be attributable to $\mathrm{CO}_{2}$ insufflation into these regions during flexible endoscopic diverticulotomy. In this patient, these findings were selflimiting and did not translate into clinical adverse events, as $\mathrm{CO}_{2}$ is spontaneously reabsorbed (16).

\section{Conclusions}

Although a relatively uncommon occurrence overall, ZD is the most frequently encountered type of esophageal diverticulum $(17,18)$. In our study of patients undergoing
Table 2 Endoscopic procedural and hospitalization specifications (total $\mathrm{n}=21$ )

\begin{tabular}{lc}
\hline Variables & Number \\
\hline Procedural success rate & $21(100 \%)$ \\
Procedure time, mean \pm SD; min & $32 \pm 12.9$ \\
Diverticulum length, median [range]; mm ( $\mathrm{n}=13)$ & $25[15-60]$ \\
Distance from incisors, mean \pm SD; cm ( $\mathrm{n}=6)$ & $17 \pm 2.3$ \\
Type of endoscopic knife & \\
Clutch Cutter & $15(71 \%)$ \\
IT2 & $1(5 \%)$ \\
Clutch Cutter \& IT2 & $1(5 \%)$ \\
SB & $3(14 \%)$ \\
SB Junior & $1(5 \%)$ \\
Endoscopic hemostatic clips used, median [range] & $4[0-6]$ \\
Immediate postoperative adverse event & $1(5 \%)$ \\
CT esophagram postoperative day \# & \\
0 & \\
1 & $6(29 \%)$ \\
Total hospital stay, mean \pm SD; days & $15(71 \%)$ \\
\hline SD, standard devation; IT2, insulated tip type & $2 \pm 1.1$ \\
\hline
\end{tabular}

SD, standard deviation; IT2, insulated tip type 2, SB, stag beetle, $\mathrm{CT}$, computed tomography

flexible endoscopic diverticulotomy for ZD, CT esophagram was used as the standard postoperative imaging modality. Both procedure-related and additional findings were identified and triaged by acuity. Our study comprehensively details postoperative radiographic findings after flexible endoscopic diverticulotomy, which are important to know for gastroenterologists, radiologists, and surgeons alike, to differentiate procedural complication from anticipated postoperative imaging changes. In addition, CT esophagram facilitates definitive exclusion of esophageal perforation as a postoperative complication of flexible endoscopic diverticulotomy by ruling out extraluminal air and contrast extravasation.

There is widespread acceptance for symptomatic patients undergoing video fluoroscopy (ex. barium esophagram) to aid in diagnosis of $Z D(2,19)$. On the other hand, there is no consensus on the optimal postprocedural imaging strategy in patients who undergo treatment of ZD. Huberty et al. described postoperative same-day barium esophagram to exclude esophageal perforation and subsequently allow oral 

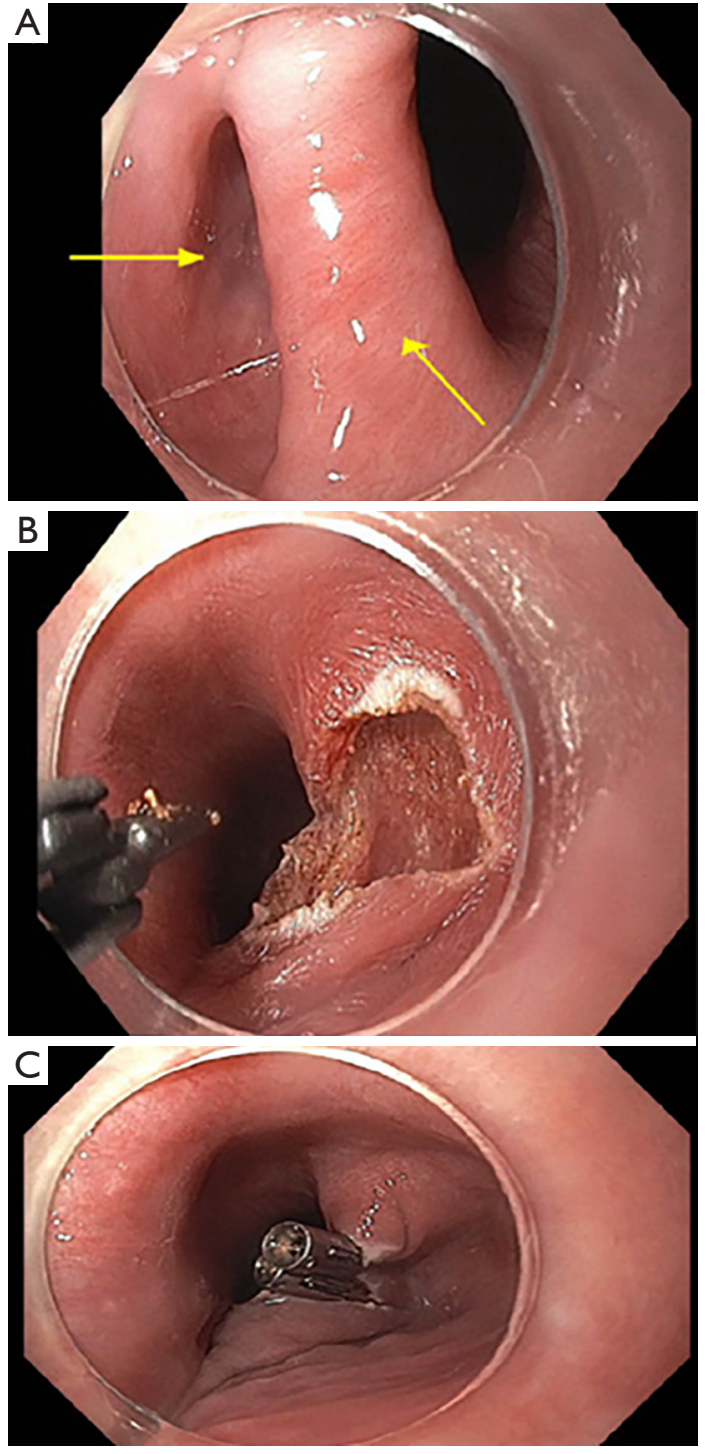

Figure 2 Endoscopic views of flexible endoscopic diverticulotomy for Zenker's diverticulum. (A) Endoscopic view of Zenker's diverticulum and septum (bottom arrow) separating true from false lumen (left arrow). (B) Endoscopic view after septotomy performed with Clutch Cutter electrosurgical scissors. (C) Completed septotomy with hemostatic clips placed to appose defect.

diet resumption (20). Other reports suggest no immediate postoperative imaging if patients remain asymptomatic, instead opting for scheduled surveillance upper endoscopy in 3 months after hospital discharge $(21,22)$. Indeed, there is no clear guideline regarding postoperative imaging even in the surgical literature describing transoral rigid stapled diverticulotomy. Dissard et al. described $16 \%$ of 50 patients obtaining a postoperative cervicothoracic CT scan in their retrospective cohort review (23).

CT esophagram has multiple advantages compared to standard video esophagography. In our institution, CT esophagram is readily available at all times-including nights and weekends-and can be performed by a CT technician without a radiologist present at the time of scan. The CT esophagram scans multiple body regions promoting detection of abnormalities in the mediastinum, lungs, and upper abdomen. Finally, utilizing both thick and thin oral contrast mediums may carry a greater sensitivity to detecting serious complications that may otherwise be missed, such as microperforations or abscesses (11). In our study, postoperative CT esophagram revealed extraluminal air and contrast extravasation consistent with esophageal perforation in a patient in whom no evidence of adverse events was detected at the conclusion of the procedure. Prompt detection allowed swift triage and intervention. Furthermore, the routine use of CT esophagram has allowed us to identify and characterize other postoperative findings that would otherwise be missed with traditional video fluoroscopy or no imaging at all. In our study, atelectasis and aspiration were seen in $62 \%$ and $10 \%$ of our patients, respectively. As a result, our postoperative protocol has expanded to include patient activity instructions to remain out of bed every two hours on the day of the procedure as well as providing an incentive spirometer for use every two hours while awake. Additionally, intravenous antibiotics are routinely scheduled while the patients remain $\mathrm{NPO}$ on the day of procedure. Once the CT esophagram excluded perforation, antibiotics were transitioned to oral equivalent formulations to complete a total five-day prophylactic course.

This study comprises several strengths. First, our series serves as the first CT esophagram protocol of $\mathrm{ZD}$ offering similar advantages to that previously described of CT esophagram after POEM (11). Second, we account for potential sources of variability. Discrepancies in initial imaging interpretations were bypassed by an unbiased radiologist independently interpreting each CT esophagram. Third, all cases described in the study met specific inclusion criteria, underwent the same flexible endoscopic procedure and completed imaging promptly by POD 1 . All patients that received flexible endoscopic therapy for symptomatic ZD also received CT esophagram and were included, therefore precluding the risk for selection bias.

Limitations include the small sample size of patients within a single institution. Nevertheless, our study had 

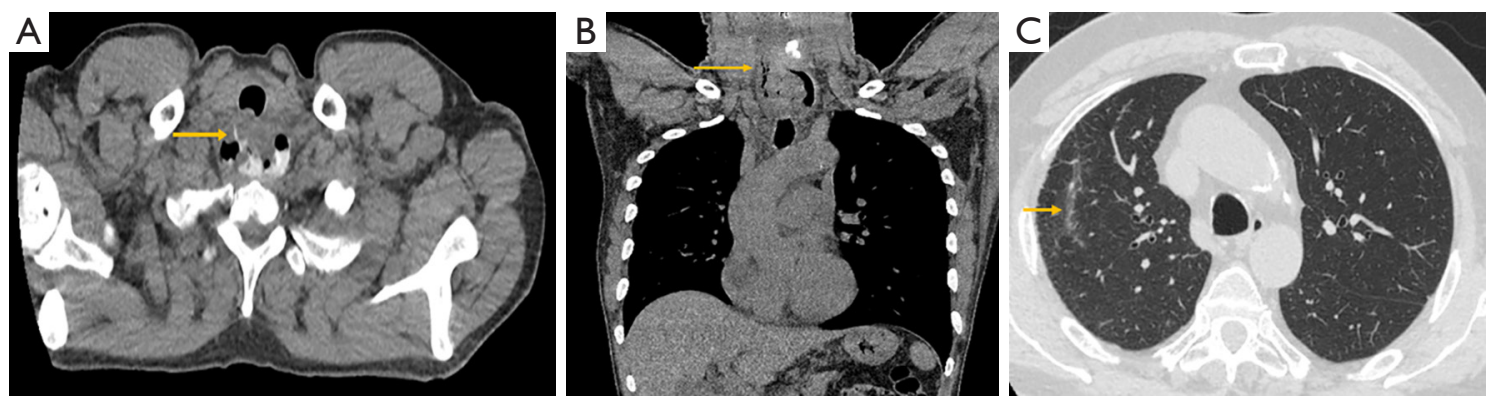

Figure 3 Representative example findings on postoperative computed tomography (CT) esophagram. (A) Axial view of CT esophagram depicting oral contrast extravasation along the right lateral border of upper esophagus (arrow). (B) Coronal view of contained esophageal perforation (arrow). (C) Axial view of right upper lobe atelectasis (arrow) without oral contrast extravasation from esophagus.

Table 3 Flexible endoscopic diverticulotomy-related findings described on postoperative CT esophagram

\begin{tabular}{|c|c|c|}
\hline Endoscopic septotomy-related findings on CT esophagram & \multicolumn{2}{|c|}{ Number $(\mathrm{N}=21)[\%]$} \\
\hline Atelectasis & $4[19]$ & 9 [43] \\
\hline Persistent diverticulum & $4[19]$ & $3[14]$ \\
\hline Pneumomediastinum & 0 & $3[14]$ \\
\hline Tree-in-bud nodularities & $1[5]$ & $2[10]$ \\
\hline Aspiration without consolidation & $1[5]$ & $1[5]$ \\
\hline Pericardial effusion & 0 & $2[10]$ \\
\hline Pleural effusion & 0 & $2[10]$ \\
\hline Pneumoperitoneum & 0 & $1[5]$ \\
\hline Pneumothorax & 0 & $1[5]$ \\
\hline Hematoma & 0 & 0 \\
\hline Hemothorax & 0 & 0 \\
\hline Esophageal intramural air & 0 & 0 \\
\hline Pneumopericardium & 0 & 0 \\
\hline
\end{tabular}

CT, computed tomography; POD, postoperative day.

a near 1:1 male to female ratio with patients at an age range classically associated with $\mathrm{ZD}$. The retrospective nature of our study precludes statistical comparison of CT esophagram to other imaging methods. Finally, a postoperative $\mathrm{CT}$ esophagram protocol can provide greater detail about both clinically significant and incidental findings but comes at an increased financial cost and radiation exposure. As a baseline comparison, a typical single contrast fluoroscopic esophagram effective radiation dose is approximately 5 milliSieverts $(\mathrm{mSv})$, whereas CT esophagram can range from $7-10 \mathrm{mSv}$ (24). Lastly, certain nonspecific incidental findings, often benign, may elicit 
Table 4 Additional findings described on postoperative CT esophagram identified by organ system

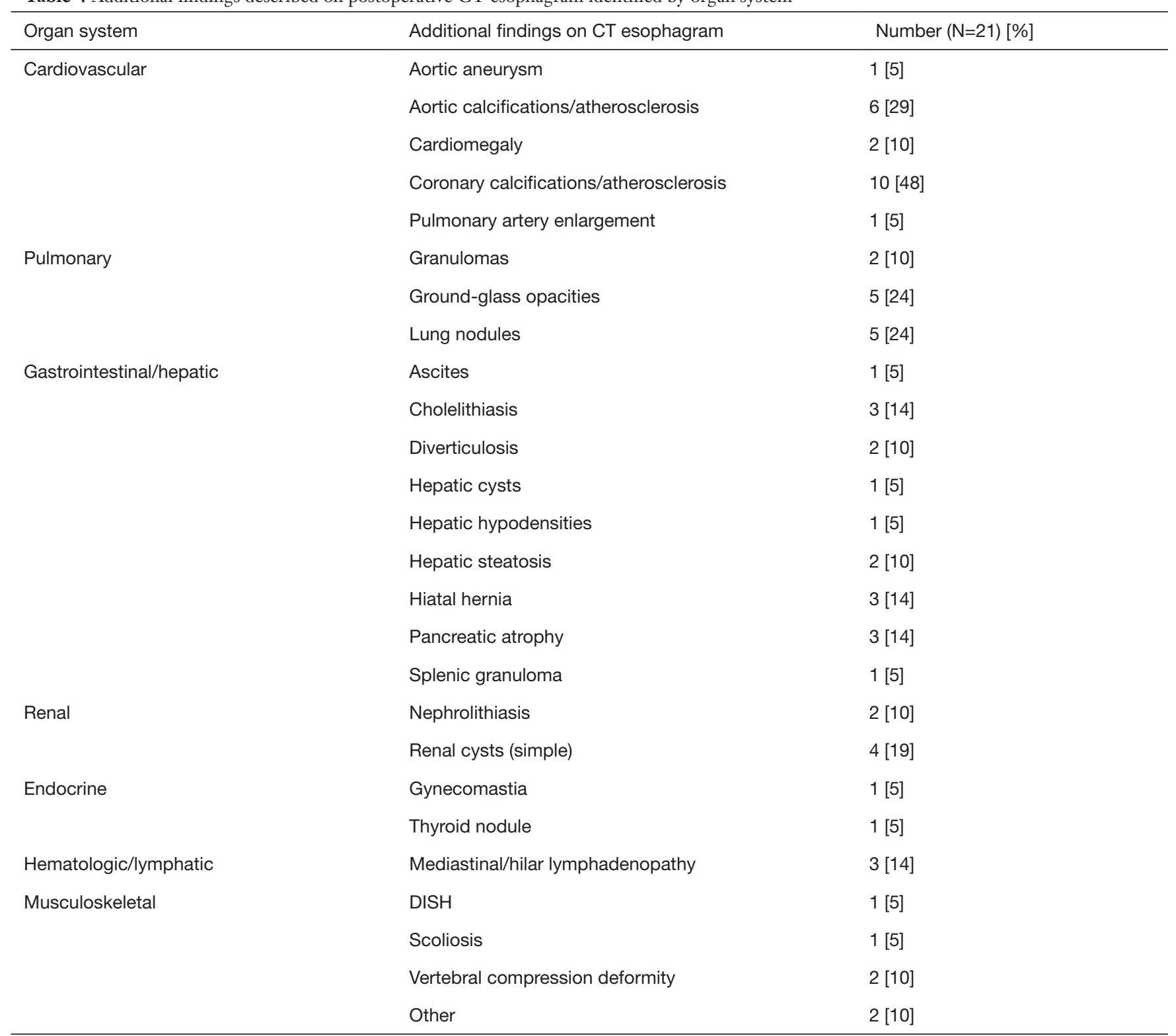

CT, computed tomography; DISH, diffuse idiopathic skeletal hyperostosis.

subsequent evaluation that may not necessarily be indicated in the absence of any associated clinical findings. The majority of the postoperative CT esophagram findings were procedure-related and self-limiting. It is plausible that the timing of the postoperative CT esophagram may have influenced a portion of these self-limiting imaging findings.

Larger studies in postoperative ZD patients with headto-head comparisons between CT esophagram and video esophagography are needed. This would allow broader application of our reported findings. It also would provide an objective comparison between the two imaging methods evaluating sensitivity and specificity for clinically significant postoperative changes. Ultimately, gastroenterologists, radiologists, and surgeons will need to tailor their respective ZD repair postoperative protocol by accounting for local comfort, expertise, and resources.

We conclude that CT esophagram expands on currently available options for postoperative imaging after flexible endoscopic diverticulotomy of ZD. The advantages of CT esophagram, including its ease of use and fidelity to describe 
postoperative detail, may prove valuable in a patient's clinical course.

\section{Acknowledgments}

The authors would like to express gratitude to Dr. Patricia L. Abbitt, MD, for her assistance with project conception and guidance in identifying key personnel for study facilitation. Funding: None.

\section{Footnote}

Reporting Checklist: The authors have completed the STROBE reporting checklist. Available at https://tgh. amegroups.com/article/view/10.21037/tgh-20-269/rc

Data Sharing Statement: Available at https://tgh.amegroups. com/article/view/10.21037/tgh-20-269/dss

Conflicts of Interest: All authors have completed the ICMJE uniform disclosure form (available at https://tgh.amegroups. com/article/view/10.21037/tgh-20-269/coif). DY reports that he is a consultant for Boston Scientific, Lumendi, and Steris, outside the submitted work; PVD reports that he is a consultant for Boston Scientific, Olympus, Cook Medical, Lumendi, and Microtech, outside the submitted work. The other authors have no conflicts of interest to declare.

Ethical Statement: The authors are accountable for all aspects of the work in ensuring that questions related to the accuracy or integrity of any part of the work are appropriately investigated and resolved. The study was conducted in accordance with the Declaration of Helsinki (as revised in 2013). The study was approved by the Institutional Review Board at the University of Florida (IRB202001012). This is a retrospective study and the Institutional Review Board does not require patients to sign informed consent forms. The study was approved as exempt as it poses minimal risk and utilized secondary research use of Identifiable Private Information for which consent is not required. All patients signed informed consent for the procedure prior to endoscopy.

Open Access Statement: This is an Open Access article distributed in accordance with the Creative Commons Attribution-NonCommercial-NoDerivs 4.0 International License (CC BY-NC-ND 4.0), which permits the noncommercial replication and distribution of the article with the strict proviso that no changes or edits are made and the original work is properly cited (including links to both the formal publication through the relevant DOI and the license). See: https://creativecommons.org/licenses/by-nc-nd/4.0/.

\section{References}

1. Beard K, Swanström LL. Zenker's diverticulum: flexible versus rigid repair. J Thorac Dis 2017;9:S154-S162.

2. Perbtani Y, Suarez A, Wagh MS. Techniques and efficacy of flexible endoscopic therapy of Zenker's diverticulum. World J Gastrointest Endosc 2015;7:206-12.

3. Hernández Mondragón OV, Solórzano Pineda MO, Blancas Valencia JM. Zenker's diverticulum: Submucosal tunneling endoscopic septum division (Z-POEM). Dig Endosc 2018;30:124.

4. Brewer Gutierrez OI, Ichkhanian Y, Spadaccini M, et al. Zenker's Diverticulum Per-Oral Endoscopic Myotomy Techniques: Changing Paradigms. Gastroenterology 2019;156:2134-5.

5. Costamagna G, Iacopini F, Bizzotto A, et al. Prognostic variables for the clinical success of flexible endoscopic septotomy of Zenker's diverticulum. Gastrointest Endosc 2016;83:765-73.

6. Sakai P. Endoscopic myotomy of Zenker's diverticulum: lessons from 3 decades of experience. Gastrointest Endosc 2016;83:774-5.

7. Sydow BD, Lebine MS, Rubesin SE, et al. Radiographic Findings and Complications After Surgical or Endoscopic Repair of Zenker's Diverticulum in 16 patients. AJR Am J Roentgenol 2001;177:1067-71.

8. Ong CC, Elton P, Mitchell D. Pharyngeal pouch endoscopic stapling--Are post-operative barium swallow radiographs of any value? J Laryngol Otol 1999;113:233-6.

9. Witterick IJ, Gullane P, Yeung E. Outcome analysis of Zenker's diverticulectomy and cricopharyngeal myotomy. Head Neck 1995;17:382-8.

10. Colombo-Benkmann M, Unruh V, Krieglstein C, et al. Cricopharyngeal myotomy in the treatment of Zenker's diverticulum. J Am Coll Surg 2003;196:370-7.

11. Pannu D, Yang D, Abbitt PL, et al. Prospective evaluation of CT esophagram findings after peroral endoscopic myotomy. Gastrointest Endosc 2016;84:408-15.

12. Buecker A, Wein BB, Neuerburg JM, et al. Esophageal perforation: comparison of use of aqueous and bariumcontaining contrast media. Radiology 1997;202:683-6.

13. Yang D, Draganov PV. Endoscopic Killian-Jamieson diverticulotomy using a scissor-type electrosurgical knife. 
Endoscopy 2018;50:E175-E176.

14. Cotton PB, Eisen GM, Aabakken L, et al. A lexicon for endoscopic adverse events: report of an ASGE workshop. Gastrointest Endosc 2010;71:446-54.

15. Ramsey D, Smithard D, Kalra L. Silent aspiration: what do we know? Dysphagia 2005;20:218-25.

16. Yang S, Zeng MS, Zhang ZY, et al. Pneumomediastinum and pneumoperitoneum on computed tomography after peroral endoscopic myotomy (POEM): postoperative changes or complications? Acta Radiol 2015;56:1216-21.

17. Shahawy S, Janisiewicz AM, Annino D, et al. A comparative study of outcomes for endoscopic diverticulotomy versus external diverticulectomy. Otolaryngol Head Neck Surg 2014;151:646-51.

18. Bresteau C, Barret M, Guillaumot MA, et al. Do we still need a diverticuloscope for the flexible endoscopic septotomy of Zenker's diverticulum? J Gastroenterol Hepatol 2020;35:630-3.

19. Tieu AH, Kumbhari V, Saxena P, et al. Flexible

doi: $10.21037 /$ tgh-20-269

Cite this article as: Banerjee D, Magnelli LL, Oliva M, Malik N, Ginsburg BM, Grajo JR, Brar TS, Westerveld DR, Yang D, Draganov PV. Characterizing a CT esophagram protocol after flexible endoscopic diverticulotomy for Zenker's diverticulum: a retrospective series. Transl Gastroenterol Hepatol 2022;7:34. endoscopic Zenker's diverticulotomy. Gastrointest Endosc 2015;81:1477.

20. Huberty V, El Bacha S, Blero D, et al. Endoscopic treatment for Zenker's diverticulum: long-term results (with video). Gastrointest Endosc 2013;77:701-7.

21. Tang SJ, Lara LF. Flexible endoscopic clip-assisted Zenker's diverticulotomy (with videos). Gastrointest Endosc 2008;67:704-8.

22. Ishaq S, Hassan C, Antonello A, et al. Flexible endoscopic treatment for Zenker's diverticulum: a systematic review and meta-analysis. Gastrointest Endosc 2016;83:10761089.e5.

23. Dissard A, Gilain L, Pastourel R, et al. Functional results in endoscopic Zenker's diverticulum surgery. Eur Ann Otorhinolaryngol Head Neck Dis 2017;134:309-13.

24. American College of Radiology ACR Appropriateness Criteria Dysphagia. ACR Appropriateness Criteria, American College of Radiology, 30 Nov. 2018. Available online: acsearch.acr.org/docs/69471/Narrative/ 